\title{
From shear bands to rapid flow: editorial
}

\author{
Scientific Conference in Monte Verità, Switzerland, February 2009
}

\author{
Dirk Kadau • Hans J. Herrmann • Sarah M. Springman • \\ Alexander Puzrin
}

Received: 25 February 2010 / Published online: 21 March 2010

(C) Springer-Verlag 2010

\section{Conference information}

Guest editor/Managing Organizer:

Dirk Kadau (ETH Zurich, Switzerland)

Organizing Committee:

Hans J. Herrmann (ETH Zurich, Switzerland)

Sarah M. Springman (ETH Zurich, Switzerland)

Alexander Puzrin (ETH Zurich, Switzerland)

Ioannis Vardoulakis (NTU Athens, Greece)

Conference web-site: http://www.shearbands.ethz.ch

D. Kadau $(\bowtie) \cdot$ H. J. Herrmann

Institute for Building Materials, ETH Zurich, Schafmattstr. 6,

8093 Zürich, Switzerland

e-mail: dkadau@ethz.ch

URL: http://www.comphys.ethz.ch

H. J. Herrmann

e-mail: hjherrmann@ethz.ch

URL: http://www.comphys.ethz.ch

S. M. Springman, A. Puzrin

Institute for Geotechnical Engineering, ETH Zurich,

Wolfgang-Pauli-Str.15, 8093 Zürich, Switzerland

e-mail: sarah.springman@igt.baug.ethz.ch

URL: http://www.igt.ethz.ch/

A. Puzrin

e-mail: alexander.puzrin@igt.baug.ethz.ch

URL: http://www.igt.ethz.ch/

\section{Theme}

Landslides and snow avalanches have plagued mountain populations for ages and have therefore been of particular interest in Swiss research activity. Indeed Switzerland does have highly sophisticated experimental installations to study avalanches at the SLF in Davos and debris flows at WSL in Davos and VAW at ETH Zurich. A key factor controlling the triggering and motion of a landslide or avalanches is the magnitude of shear stresses in the shear zone, i.e. the small region of high shear rate separating the mobilized mass from the underlying stationary ground. It can be studied from two points of view, either for geomaterials with dense packing as a shear band or for looser packings, in the more fluid-like view as rapid granular flow. For both viewpoints, either continuum theories or discrete modeling have been applied. In the last decades, the inter-disciplinarity of the topic brought together experts from different scientific disciplines and fields. However, still a common language is missing, leading to intense debates about different approaches and the validity of certain assumptions and methods. Meanwhile the theoretical quantitative prediction of all aspects of triggering, running and arrest of landsliding is not yet possible in a satisfactory way for hazard mitigation.

\section{Location}

The Centro Stefano Franscini (CSF) is the international conference center of the Swiss Federal Institute of Technology (ETH) in Zurich, situated in the south of Switzerland (Canton Ticino) at Monte Verità. It is located on the fascinating hill called the "Hill of Truth", overlooking the town of Ascona and Lake Maggiore with its Brissago Islands. This beautiful location has helped to create a relaxed atmosphere that has 


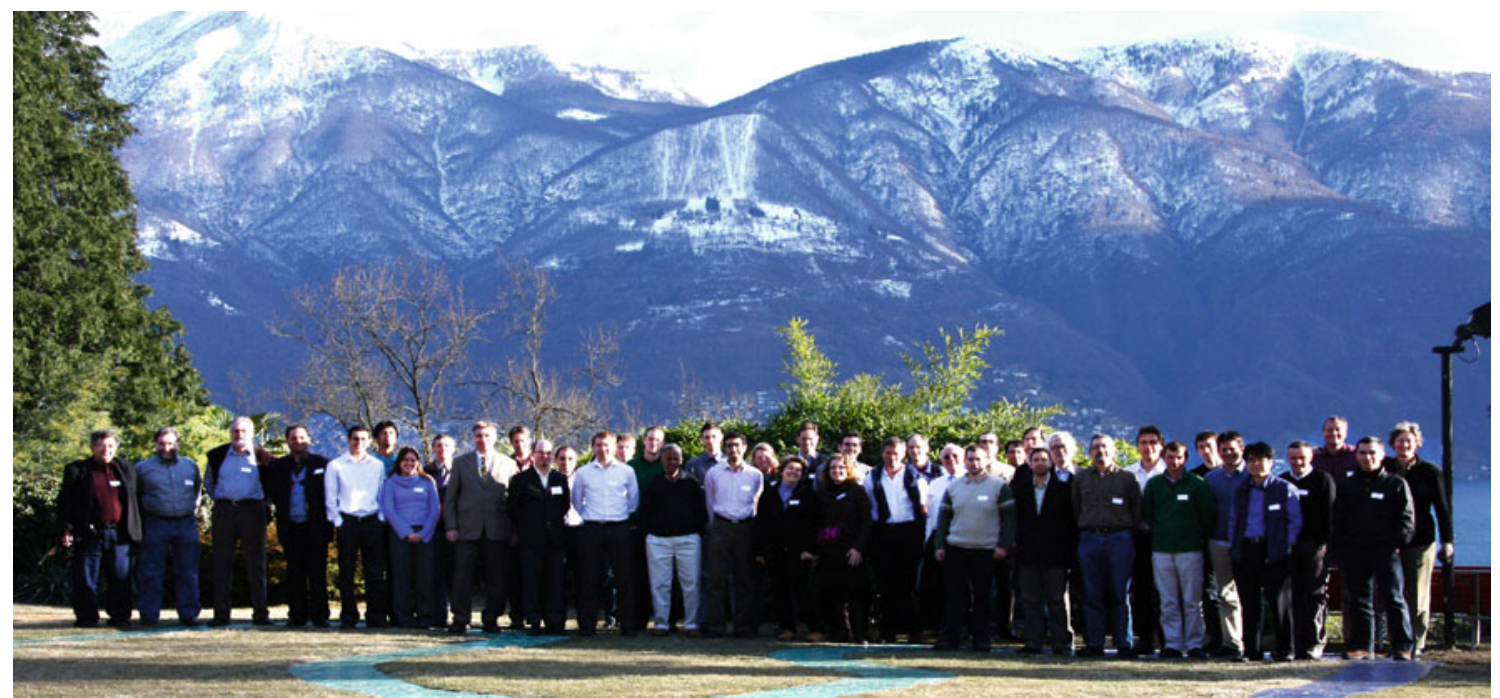

been ideally suited for intense discussion and initiation of new collaborations.

\section{Contributions to this special issue}

The workshop has been highly interdisciplinary, bringing together speakers and participants from different scientific disciplines, such as geo-engineering and physics, as well as researchers and practicians. The group also mixed together senior researchers and younger researchers most fruitfully. All of this is reflected by the selected contributions published in this special issue.

The topics of the papers are summarized in the following. Pastor et al and Muhlhaus present new insights on constitutive and rheological modeling. Goldhirsch shows how to migrate from the grain scale to continuum, Puzrin et al present a mathematical model for dynamic landslide initiation. Centrifuge technology is used for physical modeling of sturzstroms by Imre et al as well as for physical modeling of shear banding and strain softening by Röchter et al. Casini et al present laboratory tests to characterize the soil behavior at a test site. Philippe et al show an experimental granular stability analysis, whereas Schaefer et al investigate the flow behavior of a granular avalanche by detailed experimental analysis.

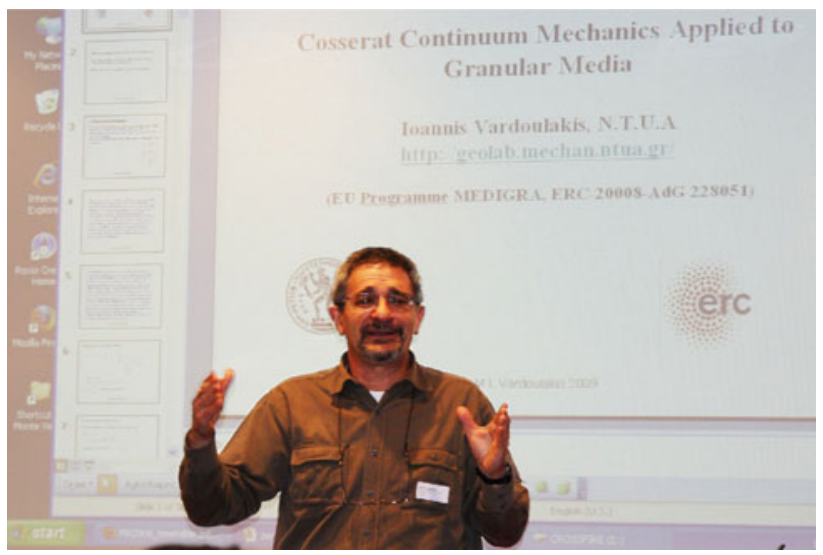

\section{Dedication}

This special issue is dedicated to the memory of our colleague and friend Professor Ioannis Vardoulakis. He was also a member of the organizing committee of this workshop. He will be remembered not only for his major contributions to the fields of geomechanics and granular mechanics, resulting in an enormous number of important publications, but also for his collegiality and positive attitude to science and life. 